\title{
Impacto da comorbidade no diagnóstico e tratamento do transtorno bipolar*
} Impact of the Comorbidity in the Diagnosis and Treament of Bipolar Disorder

\author{
RAFAel F. SANCheS ${ }^{1}$ \\ SheIlla AsSUnÇÃO ${ }^{2}$ \\ Luiz Alberto B. Hetem ${ }^{3}$
}

\begin{abstract}
Resumo
Os autores descrevem as principais comorbidades em pacientes com transtorno bipolar e suas implicações no diagnóstico e tratamento. A presença de comorbidades dificulta o diagnóstico e o manejo clínico do paciente e está associada à pior resposta ao tratamento. Dada a grande freqüência da comorbidade de transtorno bipolar com transtornos de ansiedade, é obrigatória sua pesquisa em pacientes bipolares. O tratamento do paciente bipolar com comorbidade quase sempre envolve a utilização de um estabilizador do humor. Com base nos dados de literatura não é possível dizer que um seja melhor que outro em pacientes com transtorno bipolar e comorbidade com outro transtorno. Quando se faz necessário o uso de antidepressivos há cuidados e riscos que devem ser lembrados. Os benzodiazepínicos podem ser úteis como coadjuvantes na farmacoterapia desses pacientes.
\end{abstract}

Palavras-chave: Transtorno bipolar, comorbidade, transtornos de ansiedade, diagnóstico, tratamento.

\begin{abstract}
The authors describe the main comorbidities in patients with bipolar disorder and its implications on diagnosis and treatment. The presence of comorbidities makes diagnostic procedure and clinical management of the patient more difficult and is related to poor treatment response. Due to the common cooccurrence of bipolar disorder and anxiety disorders, their presence must be considered when diagnosing and treating patients with bipolar disorder. Treatment of patients with bipolar and comorbid disorders almost always include a mood stabilizer. Based on the data available no firm recommendations can be made as to which mood stabilizer would be best for these patients. When it is necessary to use an antidepressant there are potential problems and risks that must be remembered. Benzodiazepines can be useful as coadjuvants in the pharmacotherapy of these patients.
\end{abstract}

Key words: Bipolar disorder, comorbitity, anxiety disorders, diagnosis, treatment.

Recebido: 17/11/2004 - Aceito: 07/01/2005

1 Doutorando do Programa de Pós-Graduação em Saúde Mental da Faculdade de Medicina de Ribeirão Preto da Universidade de São Paulo.

2 Médica psiquiatra. Doutora em Psiquiatria pela Faculdade de Medicina da Universidade de São Paulo.

3 Professor do Programa de Pós-Graduação em Saúde Mental da Faculdade de Medicina de Ribeirão Preto da Universidade de São Paulo.

*Apresentado no Simpósio "Diretrizes clínicas baseadas em evidências para o tratamento do transtorno bipolar", no dia 10 de agosto de 2004, em São Paulo, SP.

Endereço para correspondência: Hospital das Clínicas, Campus. Laboratório de Psicofarmacologia, $3^{\circ}$ andar - Faz. Monte Alegre - Av. Bandeirantes, 3900 - 14100-000 Ribeirão Preto - SP. Tel: (16) 602-2703; e-mail: rfsanches@uol.com.br 


\section{Introdução}

A prática clínica cotidiana com pacientes com transtorno bipolar do humor (TBH) revela que os casos de comorbidade são bastante freqüentes. Diversos estudos epidemiológicos, entre eles o Estudo Nacional de Comorbidade (National Comorbidity Survey - NCS) (Kessler et al., 1994), confirmam esse fato. Os índices de comorbidade entre pacientes com TBH variam de $30 \%$ a quase $100 \%$, conforme a metodologia e amostra selecionada (Kessler et al. 1997; Vieta et al., 2001).

As principais comorbidades em pacientes com TBH são abuso de substâncias e transtornos de ansiedade (Kessler et al., 1997). Transtornos alimentares, transtornos de personalidade e, entre outras doenças, o hipotireoidismo, a migrânea e a obesidade também são freqüentes. Estas últimas são mais comuns em mulheres do que em homens com TBH (Arnold, 2003).

Na figura 1 encontram-se as principais complicações das comorbidades. Invariavelmente, sua presença dificulta o diagnóstico e o manejo clínico do paciente com TBH e está associada a pior prognóstico, tanto em termos de resposta ao tratamento quanto de remissão (Soares et al., 2002). Por isso, sua identificação deve ser um dos pontos fundamentais em qualquer protocolo de tratamento para esses pacientes.

Nesta revisão, após descrição das principais comorbidades em pacientes com TBH, serão discutidas suas implicações clínicas e terapêuticas. Acomorbidade de TBH com abuso de substâncias, pela freqüência e peculiaridades, é apresentada no artigo "Transtorno bipolar do humor e uso indevido de substâncias psicoativas" neste suplemento (Ribeiro et al., 2005).

\section{Principais comorbidades em pacientes com TBH}

\section{Transtornos de ansiedade}

Convém insistir que comorbidade diz respeito à co- $^{-}$ ocorrência de entidades clínicas no mesmo indivíduo, ou seja, não existe comorbidade de sintomas. Isso é particularmente importante quando se trata de manifestações de ansiedade porque alguns pacientes com $\mathrm{TBH}$, notadamente aqueles em mania ou estado misto, apresentam sintomas ansiosos proeminentes. Parece até que esse alto nível de ansiedade está relacionado com pior prognóstico (Freeman et al., 2002), mas nem por isso pode-se dizer que eles tenham uma comorbidade. As verdadeiras comorbidades entre TBH e os transtornos de ansiedade são mostradas na tabela 1 - qualquer combinação entre as fases do transtorno bipolar com as dos transtornos de ansiedade é possível.

\section{Transtorno de pânico}

A comorbidade de TBH e transtorno de pânico (TP) é muito comum, tanto em estudos populacionais quanto em amostras clínicas (Freeman et al., 2002). Segundo o NCS, aproximadamente um terço dos pacientes com TBH tipo I preencheram critérios para TP. Sugere-se até a possibilidade de que os pacientes com essa comorbidade formem um subgrupo distinto de TBH (Doughty et al., 2004).

Transtorno obsessivo-compulsivo

Os resultados do Estudo Epidemiológico por Área de Captação (Epidemiologic CatchmentArea Study-ECA)

\section{Diagnóstico mais} trabalhoso

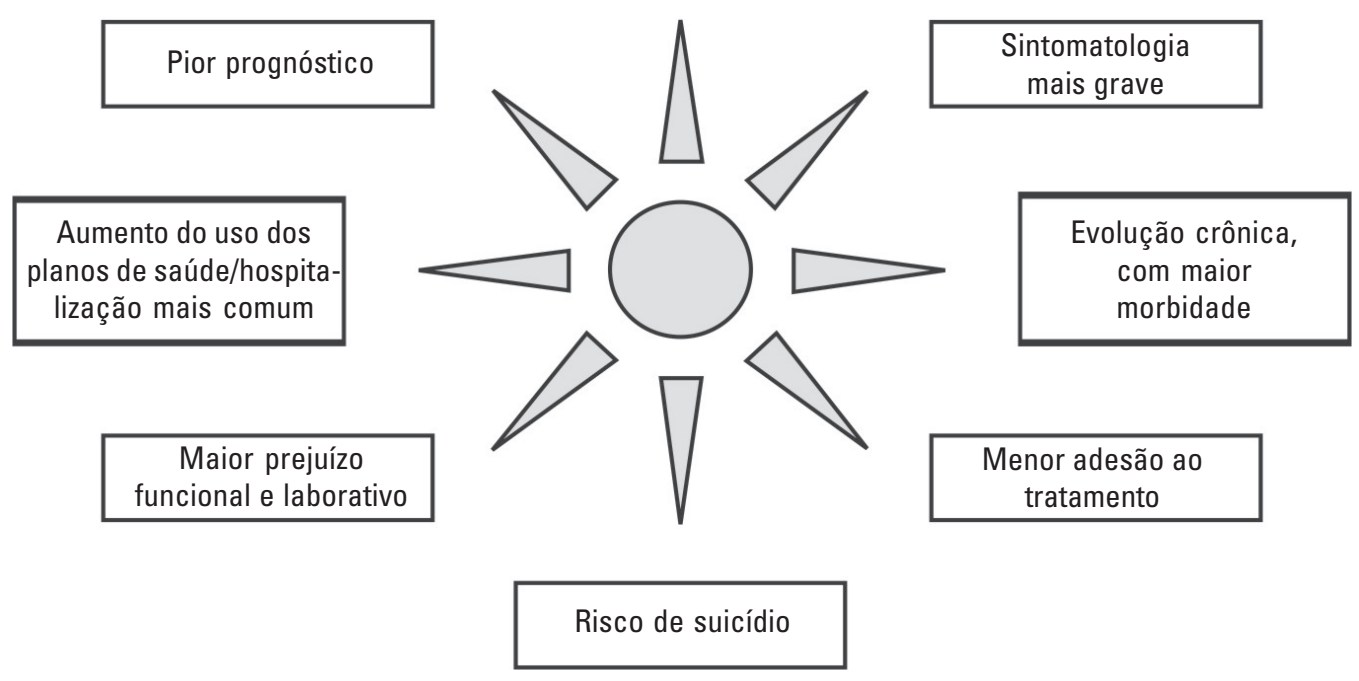

Figura 1. Complicações das comorbidades. 
Tabela 1. Comorbidades possíveis entre TBH e transtornos de ansiedade.

\begin{tabular}{l|l}
\hline Transtorno bipolar & Transtornos de ansiedade \\
\hline Hipomania/Episódio maníaco & Transtorno de pânico \\
Estado misto & Transtorno obsessivo-compulsivo \\
Depressão bipolar & Transtorno de ansiedade social \\
& Transtorno de estresse pós-traumático \\
& Transtorno de ansiedade generalizada \\
\hline
\end{tabular}

revelam a alta prevalência da comorbidade TBH com transtorno obsessivo-compulsivo (TOC), da ordem de 21\% (lifetime; Chen e Dilsaver, 1995). Em amostras clínicas esse índice pode ser até maior (Freeman et al., 2002). Esses pacientes, em comparação com os que têm somente TOC, apresentam início dos sintomas obsessivos mais gradual, com intensidade variando na dependência das oscilações de humor, maior freqüência de episódios depressivos, mais obsessões de conteúdo sexual ou religioso e menos rituais de verificação (Perugi et al., 1999).

\section{Transtorno de ansiedade social}

Embora não tão bem estudada como a comorbidade com TP e com TOC, sabe-se que a co-ocorrência de TBH e transtorno de ansiedade social (TAS) também é comum. De acordo com o NCS, aproximadamente $45 \%$ dos pacientes com TBH tipo I apresentavam também TAS (lifetime). Em geral as manifestações de TAS precedem o aparecimento dos sintomas do TBH e diminuem ou mesmo desaparecem durante as fases de excitação (hipomania ou mania) (Perugi et al., 1999).

Transtorno de estresse pós-traumático

Os dados do NCS revelam que a prevalência de TBH e transtorno de estresse pós-traumático (TEPT) comórbido é da ordem de 39\% (lifetime). A presença do TEPT provavelmente tem impacto negativo no curso e no prognóstico do TBH, visto que se trata de quadro de difícil tratamento e está freqüentemente associado com abuso de substâncias.

Transtorno de ansiedade generalizada

Diferentemente do observado em outros transtornos de ansiedade, o transtorno de ansiedade generalizada
(TAG) é mais comum em associação com depressão do que com TBH (Perugi et al., 1999).

Mais recentemente, McElroy e colaboradores (2001) estudaram as prevalências dos transtornos de ansiedade numa série de 288 pacientes com TBH, confirmando sua alta freqüência (Tabela 2).

\section{Transtornos de personalidade}

É bastante difícil o diagnóstico de transtorno de personalidade comórbido com TBH, particularmente $\mathrm{du}^{-}$ rante as fases de depressão ou de hipomania/mania. Por outro lado, sua identificação tem importantes implicações em termos de prognóstico. Assim sendo, recomenda-se que essa dúvida seja esclarecida quando o TBH estiver controlado, se possível contando com informações de familiares (Hilty et al., 1999).

Os transtornos de personalidade mais comumente encontrados em comorbidade com o TAB são os do cluster $\mathrm{B}$, principalmente os transtornos borderline e histriônico. Nesses pacientes e em outros com traços de personalidade mal adaptativa, a avaliação dos sintomas de TBH é difícil e, não raramente, seus sintomas podem ser subestimados (Soares et al. 2002).

\section{Transtornos alimentares}

Caracterizados primordialmente por distorção na percepção da imagem corporal, os transtornos alimentares - anorexia nervosa, bulimia nervosa e transtorno da compulsão alimentar periódica - estão associados a graves alterações no comportamento alimentar. Sua comorbidade com TBH também é bastante comum, principalmente nas formas mais "leves" deste quadro, como os bipolares do tipo II. McElroy et al. (2001), por exemplo, mostraram prevalência de $6 \%$ de transtorno

Tabela 2. Prevalência de transtornos de ansiedade em pacientes com TBH.

\begin{tabular}{l|c|c}
\hline & Prevalência atual (\%) & Prevalência “lifetime” (\%) \\
\hline Transtornos de ansiedade (geral) & 30 & 42 \\
\hline Transtorno de pânico & 9 & 20 \\
\hline Transtorno de ansiedade social & 13 & 16 \\
\hline Transtorno obsessivo-compulsivo & 8 & 9 \\
\hline TEPT & 4 & 7 \\
\hline TAG & 3 & 3 \\
\hline
\end{tabular}


alimentar em indivíduos com TBH. Bipolares do tipo II apresentavam maior risco de transtorno alimentar concomitante quando comparados aos do tipo I ( $12 \% \mathrm{e}$ $5 \%$, respectivamente), não havendo, no entanto, diferença na prevalência do tipo de transtorno alimentar (anorexia ou bulimia) entre os tipos I e II.

Em pacientes com bulimia nervosa, alterações de humor são freqüentemente descritas antes e depois de episódios de compulsão alimentar e comportamentos purgativos (Simpson et al., 1992).

\section{Transtorno de déficit de atenção e hiperatividade}

Observa-se, nestes últimos anos, um crescente e intenso debate sobre a relação entre o transtorno de déficit de atenção e hiperatividade (TDAH) e TBH. Alguns propõem que possa estar havendo superestimação do diagnóstico de TDAH quando muitos destes pacientes seriam, na verdade, bipolares. É difícil a distinção entre TBH e TDAH, pois sintomas como hiperatividade, distratibilidade e logorréia fazem parte do quadro clínico de ambos. Como as abordagens terapêuticas de TDAH e TBH são diferentes, essa confusão pode ter implicações muito sérias. Ainda assim, há a possibilidade de haver co-ocorrência destes dois transtornos num mesmo paciente, conforme descrito por muitos autores (Soares et al. 2002).

\section{Outras doenças}

\section{Hipotireoidismo}

Como bem mostram Soares et al. (2002), a presença de hipotireoidismo (clínico ou subclínico) em pacientes com TBH se deve, em muitos casos, à ação do lítio ou da carbamazepina sobre a função tireoidiana. Aobservação de que a carbamazepina também pode desencadear hipotireoidismo é relevante, pois muitos psiquiatras desconsideram esta possibilidade e substituem o lítio pela carbamazepina em pacientes com TBH que desenvolvem hipotireoidismo após o uso do lítio.

O hipotireoidismo se relaciona com menor resposta ao tratamento medicamentoso e parece predispor ciclagem rápida em mulheres com TBH (Arnold, 2003). Nesses casos, faz-se necessário o acompanhamento conjunto com endocrinologista.

\section{Migrânea}

Esta síndrome constituída por cefaléias periódicas, muitas vezes unilaterais, acompanhadas de náusea, vômito e perturbações sensoriais, ocorre com freqüência seis vezes maior do que o esperado pelo acaso (Arnold, 2003). O valproato de sódio pode ser uma opção nos pacientes com TBH e migrânea, visto que se mostrou eficaz no tratamento dos episódios de cefaléias.

\section{Obesidade}

A obesidade é fator de risco para o desenvolvimento de diabetes tipo 2, doença coronariana, hipertensão arterial e doenças cerebrovasculares. Daí a importância de identificá-la, tratá-la e, idealmente, preveni-la em pacientes com TBH. Da mesma forma que o hipotireoidismo, o sobrepeso e a obesidade são em geral conseqüências de efeitos colaterais dos medicamentos utilizados no tratamento do TBH, notadamente do uso continuado de antidepressivos, antipsicóticos ou lítio. As mulheres são mais suscetíveis a esse efeito colateral (Arnold, 2003). Por outro lado, uma revisão sistemática muito recente (McElroy et al., 2004) sugere enfaticamente que a coocorrência de TBH e obesidadeé maior do que a esperada em função de fatores iatrogênicos e do acaso.

\section{Diabetes}

Alterações no metabolismo da glicose têm sido associadas aos transtornos afetivos, tanto uni quanto bipolares. Cassidy et al. (1999) inclusive sugerem que episódios maníacos poderiam predispor o aparecimento de diabetes melito. A importância dessa observação reside no fato de que a detecção precoce e a intervenção terapêutica do diabetes pode minimizar o risco de aparecimento de doença vascular cerebral que, por sua vez, está associada à piora do transtorno bipolar. Além disso, medicações psicotrópicas podem aumentar o risco de desenvolvimento de diabetes, tanto direta quanto indiretamente.

\section{Impacto da comorbidade no diagnóstico e tratamento}

\section{Diagnóstico}

O diagnóstico de um transtorno psiquiátrico já é difícil em muitas ocasiões. O que dizer então do diagnóstico de uma comorbidade? Além disso, não é tarefa isenta de riscos. O primeiro deles diz respeito a se fazer um segundo diagnóstico quando, na verdade, o que o paciente apresenta não é outro transtorno e sim manifestações do próprio TBH que mimetizam outro quadro. Nunca é demais lembrar que não existem comorbidades de sintomas, mas sim de doenças ou transtornos. O segundo risco envolve o não- reconhecimento de um segundo (ou terceiro) transtorno que o paciente apresente concomitantemente com o TBH. Em outras palavras, uma coisa é o diagnóstico diferencial (Esse paciente tem TBH ou outro transtorno?) e outra são os diagnósticos associados, as comorbidades (Esse paciente tem TBH e que outros transtornos?).

Como sempre, quem paga o preço por um engano do médico assistente é o paciente. E esse preço pode ser elevado. Anão-identificação de uma comorbidade implicará resposta parcial ao tratamento (na melhor das hipóteses) com conseqüente maior morbidade e maior chance de complicações. Em contrapartida, a confusão de manifestações de TBH com sintomas de outros transtornos psiquiátricos poderá resultar em excessos terapêuticos. 
Em vista do que foi apresentado, é obrigatório, quando se faz o diagnóstico de TBH, que se investigue a presença de transtornos de ansiedade. A suspeita dessa comorbidade deve ser ainda maior nos pacientes que não estejam respondendo satisfatoriamente ao tratamento e/ou tenham problemas relacionados com abuso de álcool ou drogas, visto que essa é a complicação mais comum nesses casos (Kessler et al., 1997).

\section{Tratamento}

De modo geral, o tratamento dos casos de comorbidade é mais trabalhoso, exige conhecimento mais aprofundado de psicofarmacologia, com resultados muitas vezes frustrantes. Aadesão dos pacientes é menor, sua resposta ao tratamento não é tão boa e, conseqüentemente, a remissão é mais difícil de ser atingida.

O princípio geral do tratamento de qualquer paciente com transtorno bipolar, com ou sem comorbidade, está baseado no uso dos estabilizadores do humor. Isso implica risco de interações com antidepressivos, antipsicóticos e benzodiazepínicos, necessários em várias situações. As variadas modalidades psicoterapêuticas, bem como grupos de apoio e de auto-ajuda, também podem contribuir de modo significativo na melhoria da qualidade de vida desses pacientes, porém não dispensam a necessidade do uso dos medicamentos.

Todos os componentes de uma comorbidade devem ser alvo de tratamento. Mesmo que seja possível estabelecer seqüência cronológica para seu aparecimento, por exemplo, que num determinado paciente o transtorno de ansiedade social tenha precedido o surgimento do TBH e que ambos foram agravados por abuso de álcool, não é correto imaginar que, resolvendo o que apareceu primeiro, o resto melhore naturalmente.

\section{Transtornos de ansiedade}

Os antidepressivos, principalmente os de ação serotonérgica, são medicamentos de primeira linha no tratamento dos transtornos de ansiedade. O seu uso em pacientes com TBH entretanto, é mais complicado e exige maiores cuidados por pelo menos duas razões. Em primeiro lugar, pelo risco de indução de mania, de estado misto ou de ciclagem rápida. Mania (ou hipomania) relacionada ao uso de antidepressivos já foi relatada no curso do tratamento de todos os transtornos de ansiedade (Freeman et al., 2002). O segundo complicador é o risco de interação farmacocinética ou farmacodinâmica com os estabilizadores de humor (EH), base do tratamento farmacológico dos pacientes com TBH. Essa possibilidade de interação medicamentosa exige conhecimento psicofarmacológico aprofundado e mais atenção por parte do médico assistente.

No sentido inverso, alguns efeitos colaterais dos antipsicóticos, muitas vezes necessários no controle dos sintomas do TBH, podem agravar o quadro clínico dos transtornos de ansiedade (Perugi et al., 1999).
Os benzodiazepínicos ainda são muito utilizados no tratamento dos transtornos de ansiedade, embora cada vez mais como sintomáticos e coadjuvantes e não em monoterapia. São medicamentos seguros e bem tolerados mesmo quando usados em associação com antidepressivos, estabilizadores do humor e antipsicóticos. O alprazolam, o mais estudado deles no tratamento do TP, é também o mais problemático em pacientes com TBH pelo risco de desencadeamento de sintomas de hipomania (Freeman et al., 2002). O lorazepam se mostrou útil no tratamento de quadros de catatonia e agitação psicomotora e o clonazepam possui eficácia documentada em pacientes com TAS e TP.

O tratamento farmacológico do paciente com TBH e transtorno de ansiedade quase sempre envolve a utilização de um EH. Acredita-se mesmo que a administração de antidepressivo antes do controle das oscilações de humor pode exacerbar os sintomas do transtorno de ansiedade. Não há evidências consistentes de que algum EH tenha efeito terapêutico específico nos transtornos de ansiedade, com a possível exceção de lítio para atenuação da impulsividade do TEPT. Também não há estudos comparando a eficácia dos $\mathrm{EH}$ em pacientes com TBH e os diferentes tipos de transtornos de ansiedade. Por isso, nãoé possível fazer recomendações sobre qual seria o melhor EH com base no transtorno de ansiedade comórbido (Freeman et al., 2002). Começam a surgir evidências, entretanto, de que nos pacientes com TBH e transtornos de ansiedade a resposta ao tratamento de manutenção com anticonvulsivantes é pior (Henry et al. 2003). A sugestão de que a gabapentina poderia ser útil em pacientes com TBH e TAS ou TP comórbidos ainda precisa ser melhor investigada.

Algumas vezes, o EH é utilizado como profilático quando há indicação de antidepressivo. Nessas circunstâncias, mesmo quando o TBH está bem controlado, a introdução de um antidepressivo deve ser feita com cautela e com monitorização de sinais/sintomas de hipomania ou estado misto. Os benzodiazepínicos, com exceção feita ao alprazolam, e algumas formas de psicoterapia são alternativas aos antidepressivos em pacientes com TBH e TP, TOC ou TAS comórbidos.

A terapêutica farmacológica do TEPT, apesar de muito estudada nos últimos tempos, ainda não está bem definida. Problemas metodológicos (poucos estudos controlados, comorbidades freqüentes, maior parte dos estudos de curta duração - em transtorno com resposta terapêutica lenta - e parâmetros de melhora subjetivos) bem como as características do quadro (cujos componentes envolvem diferentes sistemas neurobiológicos) explicam esse fato. O lítio, a carbamazepina e o divalproato de sódio, entretanto, mostram-se promissores com base em relatos de caso e ensaios abertos. Seriam eles, então, os mais indicados em casos de comorbidade TBH-TEPT. O lítio parece ser a melhor opção nos casos com TBH clássico, que apresentem mania eufórica. 
A comorbidade com TOC, mais comumente encontrada em pacientes com episódios mistos do que em indivíduos com mania pura, apresenta um tratamento medicamentoso bastante complexo, com resultados nem sempre satisfatórios. Há evidências de eficácia dos estabilizadores do humor no TOC, contudo fundamentadas principalmente em relatos de casos isolados, ainda necessitando de estudos controlados (Soares et al. 2002).

Os antipsicóticos típicos e atípicos podem ajudar tanto no controle de pacientes com TOC quanto daqueles em fase de mania. Sugere-se, inclusive, que a olanzapina possa ser utilizada como estabilizador do humor. Dessa forma, estas medicações devem ser consideradas em pacientes com TOC e TBH, levandose em conta, porém, o risco de efeitos colaterais e interações medicamentosas (Freeman et al., 2002).

\section{Transtorno de personalidade}

Com relação ao tratamento do transtorno borderline, a literatura tem mostrado resultados nada satisfatórios com antipsicóticos típicos e atípicos, anticonvulsivantes e antidepressivos. No entanto, alguns estudos mostram benefícios com o valproato de sódio e a lamotrigina. A vantagem da lamotrigina parece estar no fato de ela ser efetiva tanto na dimensão afetiva, quanto na dimensão não-afetiva dos sintomas presentes no transtorno borderline (Preston et al. 2004), mas essa impressão precisa ser melhor investigada.

\section{Transtornos alimentares}

Agentes usualmente empregados no tratamento do TBH também podem ser úteis no tratamento de sintomas dos TAe vice-versa. O uso de antidepressivos, por exemplo, é eficaz no controle de algumas manifestações dos TA, tais como episódios de compulsão alimentar e de vômitos auto-induzidos (Bacaltchuk e Hay, 2003). Mais uma vez, então, é válida a regra de se associar $\mathrm{EH}$ como profiláticos de indução de mania, de estado misto e de ciclagem rápida, ocorrências que dificultariam enormemente o manejo clínico do paciente.

\section{Transtorno de déficit de atenção} com hiperatividade

O tratamento de pacientes com TBH e TDAH apresenta certas peculiaridades. O uso de estimulantes, primeira opção em crianças com TDAH, como também o de antidepressivos tricíclicos podem piorar o quadro do TBH. Inversamente, o carbonato de lítio, primeira opção em bipolar, não tem efeito terapêutico em TDAH. Segundo Hilty et al. (1999), a prioridade deve ser sempre a estabilização completa do humor, para então se proceder à terapêutica específica do TDAH.

Obesidade

Cada vez mais se acredita que a melhor forma de se lidar com esse problema é a orientação terapêutica precoce, pró-ativa, muitas vezes lançando mão de acompanhamento com nutricionista. Faz sentido pensar que $\mathrm{EH}$ que facilitem emagrecimento, como, por exemplo, o topiramato, fossem mais indicados nos casos de comorbidade de TBH com obesidade, bem como também que a sibutramina fosse segura e pudesse ser utilizada nesses casos, mas são necessários mais estudos para avaliar sua eficácia e segurança.

\section{Considerações finais e recomendações}

A comorbidade em pacientes com TBH, muito mais regra que exceção, é um fato e não um artefato devido a questões metodológicas dos estudos epidemiológicos como se chegou a pensar. Trata-se de fenômeno clínico dos mais relevantes com implicações em termos de diagnóstico, manejo clínico e prognóstico.

Recomenda-se que sempre se mantenha presente sua possibilidade, para que seja possível sua identificação precoce em pacientes com TBH. Esse cuidado é particularmente indicado nos casos de resposta insatisfatória ao tratamento ou naqueles com apresentação mais complexa, quando então a anamnese detalhada deve ser complementada por informações dadas pelos familiares.

O tratamento do paciente bipolar com comorbidade quase sempre envolve a utilização de um estabilizador do humor, quer seja como medicação primária ou como profilático quando se faz necessário o uso de antidepressivos - em pacientes com TP, TOC, TAS ou transtornos alimentares. Com base nos dados de literatura não é possível dizer que um estabilizador de humor seja melhor que outro em pacientes com transtorno bipolar em comorbidade com outros transtornos. Os benzodiazepínicos podem ser úteis como coadjuvantes na farmacoterapia desses pacientes, bem como também o emprego de técnicas de psicoterapia cognitiva e a grupoterapia. 
Referências bibliográficas

Arnold, L.M. - Gender Differences in Bipolar Disorder. Psychiat Clin N Am 26: 595-620, 2003.

BaCALtCHuk, J.; HaY, P. - Antidepressants versus Placebo for People with Bulimia Nervosa. Cochrane Database Syst Rev 4:CD003391, 2003.

Cassidy, F.; Ahearn, E.; Carrol, B.J. - Elevated Frequency of Diabetes Mellitus in Hospitalized Manic-depressive Patients. Am J Psychiatry 156: 1417-1420, 1999.

Chen, Y.W.; DILsaver, S.C. - Comorbidity of Panic Disorder in Bipolar Illness: Evidence from the Epidemiologic Catchment Area. Am J Psychiatry 152: 280-282, 1995.

Doughty, C.J.; Wells, J.E.; Joyce, P.R. et al. - Bipolar-Panic Disorder Comorbidity within Bipolar Disorder Families: a Study of Siblings. Bipolar Dis 6: 245-252, 2004.

Freeman, M.P.; Freeman, S.A.; McElroy, S.L. - The Comorbidity of Bipolar and Anxiety Disorders: Prevalence, Pychobiology, and Treatment Issues. J Affect Dis 68: 1-23, 2002.

Henry, C.; Van Den Bulke, D.; Bellivier, F. et al. - Anxiety Disorders in 318 Bipolar Patients: Prevalence and Impact on Illness Severity and Response to Mood Stabilizer. J Clin Psychiatry 64: 331-335, 2003.

Hilty, D.M.; Brady, K.T.; Hales, R.E. - A Review of Bipolar Disorder Among Adults. Psychiatr Serv50: 201-213, 1999.

KessleR, R.C.; McConagle, K.A., Zhao, S. et al. - Lifetime and 12month Prevalence of DSM-III-R Psychiatric Disorders in de United States: Results from de National Comorbidity Survey. Arch Gen Psychiatry51: 8-19, 1994.
Kessler, R.C.; Rubinow, D.R.; Holmes, C. et al. - The Epidemiology of DSM-III-R Bipolar I Disorder in a General Population Survey. Psychol Med 27: 1079-1089, 1997.

McElroy, S.L.; Kotwal, R.; Malhotra, S. et al. - Are Mood Disorders and Obesitt Related? A Review for the Mental Health Professional. J Clin Psychiatry 65: 634651, 2004.

McElroy, S.L.; Altshuler, L.L.; Suppes, T. et al. - Axis I Psychiatric Comorbidity and its Relationship to Historical Illness Variables in 288 Patients with Bipolar Disorder. Am J Psychiatry 158: 420-426, 2001.

Perugi, G.; Tonı, C.; AkISKal, H.S. - Anxious-bipolar Comorbidity. Diagnostic and Treatment Challenges. Psychiat Clin N Am 22: 565-583, 1999.

Preston, G.A.; Marchant, B.K.; ReimherR, F.W. et al. - Borderline Personality Disorder in Patients with Pipolar Disorder and Response to Lamotrigine. J Affect Disord 79(1-3): 297-303, 2004.

Simpson, S.G.; Al-Mufti, R.; Andersen, A.E. et al. - Bipolar II Affective Disorder in Eating Disorder Inpatients. J Nerv Ment Dis 180: 719-722, 1992.

Soares, M.B.M.; Moreno, D.H.; Moreno, R.A. - Comorbidades. In: Moreno, R. \& Moreno, D.H. (eds.): Transtorno Bipolar do Humor. Lemos, São Paulo: pp. 159-180, 2002.

Vieta, E.; Colom, F.; Corbella, B. et al. - Clinical Correlates of Psychiatric Comorbidity in Bipolar I Patients. Bipolar Disord 3: 253-258, 2001. 